\title{
Indicação de cardioversor desfibrilador implantável após morte súbita por fibrilação ventricular em pré-operatório de catarata: relato de caso
}

\author{
Indication of implantable cardioverter defibrillator after sudden death by \\ ventricular fibrillation in preoperative cataract: a case report
}

\author{
Livia Rossetti de Abreu e Lima ${ }^{1}$, Karoline Medeiros Dias ${ }^{2}$, Thacila Mozzaquatro ${ }^{2}$, Sergio Freitas \\ de Siqueira ${ }^{3}$, Anisio Alexandre Andrade Pedrosa ${ }^{4}$, Silvana Angelina D’Orio Nishioka ${ }^{4}$, Ricardo \\ Alkmim Teixeira ${ }^{4}$, Camila da Silva Oliveira ${ }^{5}$, Roberto Costa ${ }^{6}$, Martino Martinelli Filho ${ }^{7}$
}

\begin{abstract}
Abreu e Lima LR, Dias KM, Mozzaquatro T, Siqueira SF, Pedrosa AAA, Nishioka SAD, Teixeira RA, Oliveira CS, Costa R, Matinelli Filho M. Indicação de cardioversor desfibrilador implantável após morte súbita por fibrilação ventricular em pré-operatório de catarata: relato de caso / Indication of implantable cardioverter defibrillator after sudden death by ventricular fibrillation in preoperative cataract: a case report. Rev Med (São Paulo). 2017 jul.-set.;96(3):193-6.
\end{abstract}

RESUMO: Introdução: A fibrilação ventricular (FV) é um evento grave e fatal na maioria dos pacientes. Neste relato, descrevemos um caso de parada cardíaca durante pré-operatório de cirurgia de catarata em um paciente, com indicação subsequente de cardioversor desfibrilador implantável (CDI) para prevenção secundária de morte súbita cardíaca (MSC). Trata-se de um homem, 72 anos de idade, admitido para cirurgia de catarata com cardiomiopatia dilatada de etiologia desconhecida (DCM). O paciente apresentava hipertensão arterial, diabetes mellitus, hipotireoidismo e dislipidemia. Durante o período pré-operatório foi administrado, para realização de facectomia, colírio de tropicamida, fenilefrina e proximetacaína e, na sequência, o paciente desenvolveu FV e parada cardíaca. A parada foi revertida após 13 minutos de manobras de reanimação. O paciente foi encaminhado à Unidade de Estimulação Cardíaca de nossa instituição para avaliação. A ressonância magnética cardíaca não mostrou fibrose miocárdica e a coronariografia foi normal. Conclusão: Descrevemos um caso de FV intra-hospitalar, que acometeu paciente com DCM sem substrato anatômico arritmogênico. O mecanismo mais provável da arritmia ventricular foi hiperautomatismo induzido por estresse pré-operatório. O implante de CDI foi indicado para prevenção secundária de MSC, e afastadas causas reversíveis ou controláveis.

Descritores: Desfibriladores implantáveis; Extração de catarata; Felinefrina; Tropicamida; Estresse psicológico/cirurgia.

ABSTRACT: Introduction: Ventricular fibrillation is a potentially fatal event. We describe herein a case of cardiac arrest during preoperative of cataract surgery in a patient,

Artigo vinculado à Liga de Estimulação Cardíaca Artificial da FMUSP. Pôster apresentado no XXXIII Congresso Brasileiro de Arritmias Cardíacas, Porto Alegre, 23-26 nov. 2016.

1. Acadêmica de medicina, Faculdade de Medicina FMUSP, Universidade de Sao Paulo, Sao Paulo, SP, BR. Email: livia.rossetti@fm.usp.br.

2. Coordenadora de Pesquisa Clínica, Unidade Clinica de Estimulacao Cardiaca, Instituto do Coracao, Hospital das Clinicas HCFMUSP, Faculdade de Medicina FMUSP, Universidade de Sao Paulo, Sao Paulo, SP, BR. Email: karoline.dias@incor.usp.br, thacilam@hotmail.com.

3. Engenheiro Biomédico, Unidade Clinica de Estimulacao Cardiaca Instituto do Coracao, Hospital das Clinicas HCFMUSP, Faculdade de Medicina FMUSP, Universidade de Sao Paulo, Sao Paulo, SP, BR. Email: siqueira@incor.usp.br.

4. Médico Assistente, Unidade Clinica de Estimulacao Cardiaca, Instituto do Coracao, Hospital das Clinicas HCFMUSP, Faculdade de Medicina FMUSP, Universidade de Sao Paulo, Sao Paulo, SP, BR. Email: anisiopedrosa@uol.com.br, silvanadorio@uol.com.br, ricardo.alkmim@ icloud.com.

5. Técnica Especializada em Pesquisa, Unidade Clinica de Estimulacao Cardiaca, Instituto do Coracao, Hospital das Clinicas HCFMUSP, Faculdade de Medicina FMUSP, Universidade de Sao Paulo, Sao Paulo, SP, BR. Email: camila.oliveira@incor.usp.br.

6. Diretor, Unidade Cirurgica de Estimulacao Cardiaca, Instituto do Coracao, Hospital das Clinicas HCFMUSP, Faculdade de Medicina FMUSP, Universidade de Sao Paulo, Sao Paulo, SP, BR. Email: rcosta@incor.usp.br.

7. Diretor, Unidade Clinica de Estimulacao Cardiaca, Instituto do Coracao, Hospital das Clinicas HCFMUSP, Faculdade de Medicina FMUSP, Universidade de Sao Paulo, Sao Paulo, SP, BR. Email: martino@incor.usp.br.

Endereço para correspondência. Av. Dr. Enéas de Carvalho Aguiar, 44. Pinheiros, São Paulo, SP. CEP: 05403-900. 
referred to implantable cardioverter defibrillator (ICD) for secondary prevention of sudden cardiac death (SCD) afterwards. A 72-year-old man was admitted for cataract surgery with dilated cardiomyopathy of unknown etiology (DCM). The patient presented hypertension, diabetes mellitus, hypothyroidism and dyslipidemia. Preoperative medication consisted of eyedrops of phenylephyne, proximetacaine and tropicamide. The patient developed ventricular fibrillation (VF) and cardiac arrest right after the administration of the eyedrops. which was reverted after 13 minutes of reanimate maneuvers. The patient was referred to

\section{CASO CLÍNICO}

A.M.S, masculino, 72 anos, apresentou parada cardiorrespiratória (PCR) durante preparo intra-hospitalar para realização de facectomia, após uso tópico ocular de tropicamida, fenilefrina e proximetacaína.

Matriculado no Instituto do Coração (InCor) do Hospital das Clínicas da Faculdade de Medicina da Universidade de São Paulo (HCFMUSP) desde 2005 com diagnóstico de cardiomiopatia dilatada de etiologia desconhecida com fração de ejeção $33 \%$, insuficiência Cardíaca Classe Funcional I pela New York Heart Association (NYHA) e com as seguintes comorbidades associadas: hipertensão arterial sistêmica (HAS), diabetes mellitos tipo II (DM2), dislipidemia (DLP) e hipotireoidismo; sorologia para doença de Chagas negativa. Em uso de carvedilol, enalapril, glibenclamida, metformina, atorvastatina, levotiroxina e AAS, compensado clinicamente e com provas tireoideanas (T4 e TSH) normais.

Assistido no ambulatório da Cirurgia de Oftalmologia do HCFMUSP em fibrilação ventricular (FV) seguida de atividade elétrica sem pulso (AESP), tendo sido submetido a manobras de reanimação durante 13 minutos, com sucesso na reversão do ritmo.

Encaminhado ao pronto socorro do InCor sob suspeita de FV induzida por administração de medicação tópica oftalmológica. Admitido em uso de noradrenalina e drogas vasoativas (DVA), sob intubação orotraqueal (IOT) em ventilação mecânica (VM), monitorização eletrocardiográfica com pressão arterial (PA) 100x60 $\mathrm{mmHg}$, eletrocardiograma de 12 derivações (ECG 12d) demonstrando ritmo sinusal com bloqueio de ramo esquerdo, extra-sístoles ventriculares frequentes e sem sinais de isquemia aguda. No dia seguinte, ainda sob IOT, com desmame de DVA e com suspensão da noradrenalina ao exame físico apresentava frequência respiratória (FR) de 15-19 ipm, frequência cardíaca (FC) de 74-88 bpm, pressão arterial sistólica $128-154 \mathrm{mmHg}$ e pressão arterial diastólica 64-78 mmHg, bulhas hipofonéticas, sem sopros. Aos exames laboratoriais apresentava apenas alteração da proteína $\mathrm{C}$ e enzimas cardíacas. A cinecoronariografia não demonstrou lesões obstrutivas coronarianas.

Paciente evoluiu bem, sendo transferido para a the Cardiac Pacing Unit of our institution for evaluation. Cardiac MRI showed no myocardial fibrosis and coronary angiography was normal. Conclusion: We describe a case of in-hospital VF, in a patient without arrhythmogenic anatomical substrate. The most likely mechanism of ventricular arrhythmia was hyper automatism induced by preoperative stress. The ICD was implanted for secondary prevention $\mathrm{SCD}$, considering controllable or reversible causes were ruled out.

Keywords: Defibrillators, implantable; Cataract extraction; Phenylephrine; Tropicamide; Stress psychological/surgery.

enfermaria. Não apresentou intercorrências, mantendo estabilidade hemodinâmica e boa função renal. Os níveis de CPK permaneceram elevados, mas foi descartada rabdomiólise. O ecocardiograma revelava fração de ejeção do ventrículo esquerdo (FEVE) de 30\%, semelhante aos achados de um ano antes. Ressonância magnética cardíaca (RMC) demonstrava ausência de realce tardio miocárdico.

Paciente realizou implante de Cardioversor Desfibrilador Implantável (CDI) como prevenção secundária de morte súbita cardíaca (MSC) e evoluiu com estabilidade clinica e função renal preservada, tendo recebido alta hospitalar no dia seguinte. Apresentando-se em consulta um mês após a cirurgia assintomático do ponto de vista clínico, sem terapias no CDI. Avaliação eletrônica.

\section{DISCUSSÃO}

Descreve-se um caso de FV ocorrida em ambiente hospitalar em portador de cardiomiopatia dilatada (CMD), apresentando hipocaptação persistente inferior e apical em cintilografia miocárdica prévia mas sem evidência de substrato anatômico arritmogênico à investigação com ecocardiograma transtorácico, condição predominante para a ocorrência de eventos fatais em cardiopatias adquiridas.

A CMD é uma patologia primária progressiva que leva a dilatação ventricular, mais frequentemente do ventrículo esquerdo (VE), com disfunção sistólica ${ }^{1}$, redução da contratilidade cardíaca ${ }^{2}$ e diminuição da fração de ejeção (FE). Pacientes em fase inicial podem ser assintomáticos, mas em fases avançadas podem ocorrer congestão pulmonar, arritmias cardíacas, anormalidades do sistema de condução do coração e tromboembolismo arterial.

Estudos recentes nas áreas de imunologia, genética, biologia celular e molecular ${ }^{3}$ apontam para o caráter multifatorial da doença atualmente descrita como sendo uma resposta miocárdica a fatores genéticos e ambientais ${ }^{1}$. A maioria dos casos não apresenta etiologia definida, sendo classificados como idiopáticos. Entre as causas definidas estão alterações genéticas (CMD congênita), toxinas, distúrbios endocrinológicos, patologias neuromusculares, moléstias inflamatórias, infecciosas e autoimunes. $\mathrm{Na}$ sua fisiopatologia, devem ser considerada a sobreposição 
de fatores indutores de remodelação ventricular, como hipertensão arterial sistêmica (HAS), isquemia miocárdica e cirrose hepática.

A variabilidade de critérios diagnósticos dificultam inferências estatísticas ao redor do mundo. Em 2004, estudos europeus e norte-americanos citavam incidência geral de 2 a 8 casos por 100.000 habitantes e uma prevalência de 36 casos por 100.000 habitantes ${ }^{4}$.

Atualmente a CMD é uma das principais causas de insuficiência cardíaca congestiva (ICC), com indicação de transplante cardíaco em jovens. A CMD está intimamente relacionada à morte súbita por arritmia ventricular maligna.

A FV é uma atividade elétrica turbulenta, resultante da propagação de múltiplas ondas independentes e aperiódicas ${ }^{5}$ em todo o músculo cardíaco, geralmente desencadeada a partir de taquicardia ventricular. Atualmente acredita-se que um terço das mortes súbitas cardíacas sejam decorrentes da FV.

Hiperautomatismo ventricular (excitabilidade exacerbada tecidual), atividade deflagrada (potenciais tardios) ou circuitos de reentrada ${ }^{6}$ têm sido descritos como principais substratos da FV. Em geral, os pacientes acometidos apresentam ${ }^{7}$ alterações cardiacas estruturais constituídas por tecido isquêmico, inflamatório e fibrose miocárdica. Atualmente considera-se que FE VE reduzida e a insuficiência cardíaca são os mais importantes fatores de risco para morte súbita por arritmia nesses pacientes

No caso descrito, a FV parece estar associada ao mecanismo de hiperautomatismo. Esta conclusão é corroborada pelo ECG 12d que demonstrou Intervalo QT normal e a RMC não revela sinais inflamatórios ou presença de fibrose miocárdica. Nesse sentido, vários fármacos foram descritos como possíveis indutores de taquiarritmias ventriculares, incluindo os de uso tópico ${ }^{8,9,10}$.

Inúmeras drogas ${ }^{8}$ estão disponíveis para aplicação tópica em procedimentos oftalmológicos, a maioria delas atuando sobre receptores colinérgicos e adrenérgicos. Sua administração não é livre de efeitos colaterais sistêmicos e estes podem ocorrer em função da superdose, comorbidades do paciente ou interações com outras drogas, em geral anestésicos locais ou gerais. Dentre estas, destacamos:

\section{Tropicamida}<smiles>CCN(Cc1ccncc1)C(=O)C(CO)c1ccccc1</smiles>

Parassimpatolítico (anticolinérgico muscarínico direto). Atua bloqueando os receptores de acetilcolina e promovendo o relaxamento dos músculos do esfíncter pupilar, utilizado para indução de midríase e cicloplegia. Contra indicado para pacientes portadores de glaucoma de ângulo fechado.

\section{Fenilefrina}<smiles>CNC[C@H](O)c1cccc(O)c1</smiles>

A fenilefrina age como agonista seletivo alfa1, utilizado como indutor tópico de midríase (solução 10\%), descongestionante nasal, de trompas de Eustáquio e agente cardiotônico 9 .

Estudo prospectivo ${ }^{10} \mathrm{em} 2009$, não demonstrou elevação estatisticamente significativa da pressão arterial sistêmica em $87 \%$ de normotensos (55) e $76 \%$ dos hipertensos (34) estudados, após aplicação de fenilefrina tópica a $10 \%$. Apenas um paciente apresentou aumento significativo da pressão arterial, sugerindo que sua utilização é segura ${ }^{10,11}$. A reação adversa estaria relacionada a superdose ou idiossincrasia individual, e a interação com outros fatores não foi elucidada.

Crise hipertensiva, taquicardia e bradicardia reflexa, hemorragias subaracnóideas, arritmias ventriculares, infarto do miocárdio e parada cardíaca estão entre os efeitos adversos raros relatados na literatura ${ }^{8,12}$.

Em 1978, publicação ${ }^{12}$ do Americal Journal of Ophthalmology apontava existirem 33 reações graves possivelmente relacionadas à aplicação de fenilefrina tópica a 10\%, reportadas ao "Registro Nacional Efeitos Colaterais Induzida por Drogas de Uso Ocular", do Departamento de Oftalmologia da Universidade do Arkansas, EUA (National Registry of Drug-Induced Ocular Side Effects, Department of Ophthlamology, University of Arkansas for Medical Sciences, Little Rock, Arkansas).

\section{Proximetacaína}<smiles>CCCOc1ccc(C(=O)OCCN(CC)CC)cc1N</smiles>

Anestésico local de ação rápida para uso oftalmológico que promove grande aumento transitório na permeabilidade da membrana para íons de sódio. Deve ser utilizado com cautela em pacientes hipertensos e portadores de hipertireoidismo.

No caso descrito foram administrados os fármacos tópicos tropicamida, e proximetacaína que, conforme descrito acima, não parecem estar associados às situações emergenciais vivenciadas e a fenilefrina, que dependendo 
da dosagem e da interação de outros fatores pode estar implicada.

Finalmente, é importante destacar que a deflagração das ocorrências do caso descrito pode estar associada ao estresse peri-operatório. Este inclui as situações vivenciadas no ambiente hospitalar, proximidade temporal da anestesia e cirurgia que podem alterar significativamente os níveis de estresse emocional dos pacientes, provocar expressiva liberação catecolaminérgica e desencadear taquiarrimias fatais. Ademais, um estudo ${ }^{13}$ demonstra que o stress perioperatorio é expressivamente influenciado pela comunicação efetiva da equipe, contato pré-admissional, ambiente de espera, privacidade e fornecimento de informações específicas ${ }^{13,14,15,16}$.

Sumarizando, o caso descrito de FV ocorrida em ambiente hospitalar em portador de cardiomiopatia dilatada (CMD), sem evidência de substrato anatômico arritmogênico, tem como provável mecanismo o hiperautomatismo ventricular, possivelmente induzido por estresse peri-operatório. Nesse sentido, o implante de CDI é imperioso para prevenção secundaria de MSC, não associada a causas reversíveis ou controláveis.

Agradecimento especial: Dr. Martino Martinelli Filho por todo o incentivo e aprendizado.

\section{REFERÊNCIAS}

1. Japp AG, Gulati A, Cook SA, Cowie MR, Prasad SK. The diagnosis and evaluation of dilated cardiomyopathy. J Am Coll Cardiol. 2016;67(25):2996-3010. doi: 10.1016/j. jacc.2016.03.590.

2. Hershberger RE, Morales A. Dilated cardiomyopathy overview. 2007 Jul 27 [Updated 2015 Sep 24]. In: Pagon RA, Adam MP, Ardinger HH, et al., editors. GeneReviews ${ }^{\circledR}$. Seatle (WA): University of Washington; 1993-2017. Available from: https:/www.ncbi.nlm.nih.gov/books/NBK1309/.

3. Mattos BP. Mecanismos celulares e biomoleculares na cardiomiopatia dilatada. Arq Bras Cardiol. 1999;72(4):5125. Disponível em: http://publicacoes.cardiol.br/ abc/1999/7204/72040011.pdf.

4. Horowitz ESK. Miocardiopatia dilatada: manejo clínico. Rev Soc Cardiol Rio Grande do Sul. 2004;13(1):1-4. Disponível em: http://sociedades.cardiol.br/sbc-rs/revista/2004/01/ artigo09.pdf.

5. Cakulev I, Efimov IR, Waldo AL. Cardioversion: past, present, and future. Circulation. 2009;120(16):1623-32. doi: 10.1161/ CIRCULATIONAHA.109.865535

6. Samie FH, Jalife J. Mechanisms underlying ventricular tachycardia and its transition to ventricular fibrillation in the structurally normal heart. Cardiovasc Res. 2001;50:242-50.

7. Dosdall DJ, Ideker RE. Mechanisms of ventricular tachycardia and fibrillation. In: Cardiac electrophysiology: from cell to bedside. 6th ed. Atlanta: Elsevier; 2013. p.47582. doi: 10.1016/B978-1-4557-2856-5.00048-0.

8. Gunaydin B, Cok OY. Hazards of topical ophthalmic drug administration. Trends Anaesth Crit Care. 2011;1(1):31-4. doi: 10.1016/j.cacc.2010.07.001.
9. Stavert B, McGuinness MB, Harper C, Guymer RH, Finger RP. Cardiovascular adverse effects of phenylephrine eyedrops: a systematic review and meta-analysis. JAMA Ophthalmol. 2015;133(6):647-52. doi: 10.1001/ jamaophthalmol.2015.0325.

10. Bhatia J, Varghese M, Bhatia A. Effect of $10 \%$ phenylephrine eye drops on systemic blood pressure in normotensive \& hypertensive patient. Oman Med J. 2009;24(1):30-2. doi: 10.5001/omj.2009.8.

11. Kumar V, Schoenwald RD, Chien DS, Packer AJ, Choi WW. Systemic absorption and cardiovascular effects of phenylephrine eyedrops. Am J Ophthalmol. 1985;99(2):1804.

12. Fraunfelder FT, Scafidi AF. Possible adverse effects from topical ocular 10\% phenylephrine. Am J Ophthalmol. 1978;85(4):447-53.

13. Rhodes L, Miles G, Pearson A. Patient subjective experience and satisfaction during the perioperative period in the day surgery setting: a systematic review. Int J Nurs Pract. 2006;12:178-92. doi: 10.1111/j.1440-172X.2006.00575.x.

14. Davis Y, Perham M, Hurd AM, Jagersky R, Gorman WJ, Lynch-Carlson D, Senseney D. Patient and family member needs during the perioperative period. J Perianesth Nurs. 2014;29(2):119-28. doi: 10.1016/j.jopan.2013.05.013.

15. Suominen T, Turtiainen A-M, Puukka P, Leino-Kilpi H. Continuity of care in day surgical care - perspective of patients. Scand J Caring Sci. 2014;28(4):706-15. doi: 10.1111/ scs. 12099.

16. Tiwari W, Queenan C, Jacques PS. Impact of waiting and provider behavior on surgical outpatients' perception of care. Perioperative Care Operating Room Manag. 2017;7:7-11. https://doi.org/10.1016/j.pcorm.2017.03.002. 